\title{
Influence of sampling interval on the evaluation of nocturnal blood pressure in subjects with and without obstructive sleep apnoea
}

\author{
O. Marrone, S. Romano, G. Insalaco, M.R. Bonsignore, A. Salvaggio, G. Bonsignore
}

\begin{abstract}
Influence of sampling interval on the evaluation of nocturnal blood pressure in subjects with and without obstructive sleep apnoea. O. Marrone, S. Romano, G. Insalaco, M.R. Bonsignore, A. Salvaggio, G. Bonsignore. (C)ERS Journals Ltd 2000.

ABSTRACT: Blood pressure (BP) variability during sleep is high in obstructive sleep apnoea syndrome (OSAS). How BP sampling interval affects the estimate of mean nocturnal BP in OSAS and control subjects was investigated.

Nine subjects with apnoea/hypopnoea index (AHI) $<5$ and 18 OSAS patients with AHI $>30$ underwent nocturnal polysomnography with beat-by-beat BP monitoring. Mean nocturnal BP was evaluated averaging: a) all systolic $\left(P_{\mathrm{s}}\right)$ and diastolic $\left(P_{\mathrm{d}}\right) \mathrm{BP}$ values; b) $P$ s and $P$ d sampled every 5, 10, 15, 20, and 30 min. The sampling starting point was repeatedly shifted, and several mean BP estimates for each sampling interval were obtained. Differences $(\Delta P$ s and $\Delta P d)$ between means obtained by sampling $B P$ and by averaging all $B P$ values were calculated.

In both groups $\Delta \boldsymbol{P}_{\mathrm{s}}$ and $\Delta \boldsymbol{P}_{\mathrm{d}}$ scatter increased as sampling interval increased; their variance was always higher in OSAS subjects $(p<0.001)$. Over $95 \%$ of $\Delta P$ s and $\Delta P d$ were $<\mathbf{5 \%}$ of the beat-by-beat mean values at all sampling intervals in controls, but this occurred only at sampling intervals $\leq 10 \mathrm{~min}$ in OSAS subjects.

To conclude, for each blood pressure sampling time, a larger number of inaccurate nocturnal mean blood pressure estimates are obtained in obstructive sleep apnoea syndrome than in control subjects. Obstructive sleep apnoea syndrome subjects require more frequent blood pressure measurements to obtain a similar accuracy in nocturnal blood pressure evaluation.
\end{abstract}

Eur Respir J 2000; 16: 653-658.

Istituto di Fisiopatologia Respiratoria del CNR, Palermo, Italy.

\section{Correspondence: O. Marrone}

Istituto di Fisiopatologia Respiratoria del CNR

Via Trabucco, 180

90146 Palermo

Italy

Fax: 390916882165

Keywords: Accuracy

blood pressure monitoring

methodology

Received: November 151999

Accepted after revision June 182000
Twenty-four-h blood pressure (BP) assessment is usually performed by ambulatory blood pressure monitoring (ABPM), which allows repeated BP measurements at regular intervals. Comparison of beat-to-beat BP measurements and sampled measurements was proved reliable in hypertensive subjects unselected for sleep problems [1]. In particular, nocturnal BP measurements taken every 30 min accurately reflected mean nocturnal BP calculated with continuous BP monitoring [2]. In addition, mean nocturnal BP did not differ if calculated on measurements taken every 15 or every $60 \mathrm{~min}$ [3].

Obstructive sleep apnoea syndrome (OSAS) is a condition characterized by the recurrence of episodes of upper airway obstruction during sleep, which are usually terminated by an arousal with heavy snoring, hyperventilation, and a sudden transient rise in heart rate and BP [4]. In addition to the repeated nocturnal BP augmentations, OSAS is often associated with diurnal hypertension [4]; available data about reversibility of systemic hypertension by long-term OSAS treatment are still contradictory [5-11]. Cardiovascular accidents, possibly linked to either diurnal or nocturnal high BP, are considered common complications of this syndrome and possible causes of death [12]. A correct evaluation of BP in OSAS could help to identify subjects at highest risk of cardiovascular complications and to better understand the effect of OSAS treatment on BP. However, in patients with OSAS the occurrence of apnoeas during sleep increases nocturnal BP variability [13], and in particular its short-term variability [14]. Therefore intervals between BP measurements as long as 30 or $60 \mathrm{~min}$, commonly used for the assessment of mean nocturnal BP, could not be enough to warrant a correct assessment of mean nocturnal BP in these patients.

In this study, how the assessment of mean nocturnal BP is affected by different BP sampling rates in OSAS and in control subjects was evaluated.

\section{Patients and methods}

Eighteen patients, aged 47.6 \pm 9.3 (mean \pm SD) yrs, referred to our sleep laboratory for a strong suspicion of OSAS, and nine healthy subjects, aged $45.3 \pm 8.8$ yrs were studied.

A standard nocturnal polysomnography was performed using a computerized system (Somnostar 4100; Sensormedics, Yorba Linda, CA, USA). At the same time BP was continuously monitored by a 2300 Finapres ${ }^{\mathrm{TM}} \mathrm{BP}$ monitor (Ohmeda, Louisville, CO, USA). This device works with a dynamic servo set-point adjuster, frequently checking the 
set-point during short interruptions of the BP recording in an automatic fashion [15]. The Finapres ${ }^{\mathrm{TM}}$ was automatically turned off for $5 \mathrm{~min}$ every $40 \mathrm{~min}$ to avoid finger discomfort and local vascular or fluid changes that could alter estimation of BP. The BP signal was recorded on tape (TEAC XR-5000, Japan).

Sleep was staged according to standard rules [16]. Total time in bed (TIB), total sleep time (TST) and sleep efficiency (total sleep time/time in bed $\times 100$ ) were calculated. Apnoeas were considered as cessation of oronasal airflow lasting at least $10 \mathrm{~s}$; hypopnoeas as reduction in the airflow signal lasting at least $10 \mathrm{~s}$ associated to $\mathrm{a} \geq 3 \%$ oxyhaemoglobin saturation $\left(\mathrm{Sa}, \mathrm{O}_{2}\right)$ fall or followed by an arousal [16]. Apnoea plus hypopnoea frequency was calculated both per hour of sleep (AHI) and per hour of time in bed (AH/TIB).

The BP signal was acquired at a sampling rate of 200 $\mathrm{Hz}$ on a personal computer for off-line analysis. A program of analysis was developed using the Matlab software (Math Works Inc., Natick, MA, USA). After visual inspection of the BP signal to eliminate artefacts, systolic and diastolic BP values, in $\mathrm{mmHg}$, were obtained, and mean nocturnal systolic $(P \mathrm{~s})$ and diastolic $(P \mathrm{~d}) \mathrm{BP}$ values were calculated: a) on all the recorded nocturnal heart beats; $b$ ) on single heart beats sampled every $5,10,15,20$, and 30 min. Mean values calculated averaging all nocturnal heart beats were considered "real" reference values, while mean values calculated averaging the single sampled heart beats were considered as "estimates". To account for effects of different starting points in the sampling, the starting point was shifted several times by one minute for every sampling interval so as to obtain, for each subject, up to $5,10,15,20$, and 30 mean estimated BP values, respectively, for the different sampling intervals (fig. 1). Due to the occurrence of autocalibrations, temporary turn-offs or artefacts on the Finapres ${ }^{\mathrm{TM}}$ signal, the number of points actually sampled for the calculation of some mean values had to be lower than expected; a threshold of a minimum of $60,30,20$, 15 , and 10 points for the sampling intervals of $5,10,15$,

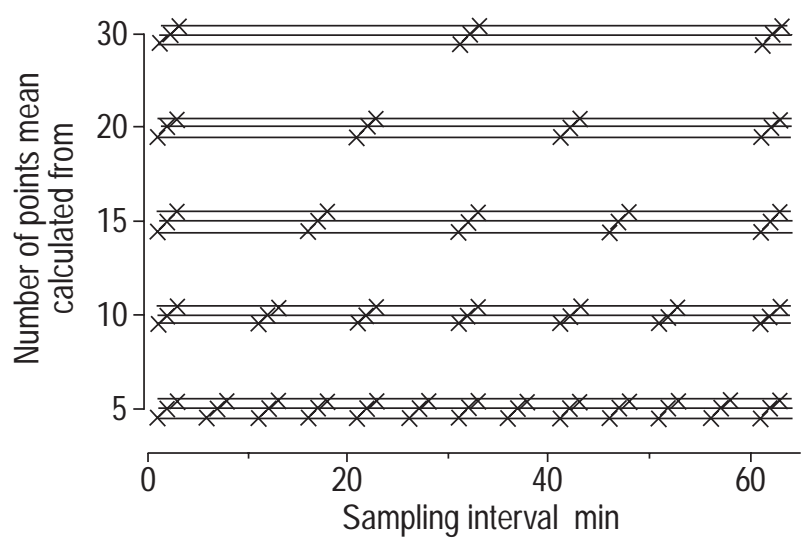

Fig. 1. - Schematic representation of the method used to sample blood pressure values. Each horizontal line represents the same series of all heart beats recorded during the first hour of the nocturnal monitoring in one patient. Symbols x represent one heart beat, for which systolic and diastolic blood pressures were measured. The first $x$ on each line represents the first heart beat from which the sampling was started. In all subjects, the sampling starting point was shifted several times by $1 \mathrm{~min}$ : in this way, depending on the sampling interval, up to 30 sets of systolic pressure $(P \mathrm{~s})$ and diastolic pressure $(P \mathrm{~d})$ values were collected to calculate "estimates" of mean nocturnal $P \mathrm{~s}$ and $P \mathrm{~d}$.
20, and 30 minutes, respectively, for a mean BP estimate to be accepted was established. In all subjects and for all sampling intervals the number of mean nocturnal BP estimates and the number of points averaged for each estimate was always the same for systolic and diastolic values.

To evaluate the error due to each sampling interval, the following values were calculated:

$\Delta P_{\mathrm{s}}=$ mean estimated $P_{\mathrm{s}}-$ mean real $P_{\mathrm{s}}$

$\Delta P \mathrm{~d}=$ mean estimated $P \mathrm{~d}-$ mean real $P \mathrm{~d}$

$\Delta P \mathrm{~s} \%=($ mean estimated $P \mathrm{~s}-$ mean real $P \mathrm{~s}) /$ mean real $P_{\mathrm{s}}$ $\times 100$

$\Delta P \mathrm{~d} \%=($ mean estimated $P \mathrm{~d}-$ mean real $P \mathrm{~d} /$ mean real $P \mathrm{~d} \times 100$

Coefficients of variation of beat-by-beat $P \mathrm{~s}$ and $P \mathrm{~d}$ were calculated for every normal and OSAS subject. Mean \pm SD of $\Delta P \mathrm{~s}$ and $\Delta P \mathrm{~d}$ were calculated separately for the OSAS population and for the controls for each sampling interval. Differences in variance in $\Delta P \mathrm{~s}$ and $\Delta P \mathrm{~d}$ values at each sampling interval between the control and the OSAS group were evaluated by the F test. Differences in other variables were tested by two-tailed unpaired t-test or U-Mann Whitney test. Correlations between variables were evaluated by linear regression analysis. A $p<0.0 .5$ was considered significant.

\section{Results}

Control and OSAS groups did not differ in TIB and TST, while sleep efficiency was slightly, but significantly, higher in the OSAS group. AHI was on average very high in the OSAS group, although with a large range (47-144). Real mean BP values were higher in the OSAS subjects than in controls (table 1).

The number of mean nocturnal BP estimates and the number of points used for the calculations are shown in table 2. At all sampling intervals, there was no difference between groups in the mean number of points averaged per mean BP estimate or in the average number of mean BP estimates per subject. There was no correlation between the number of points used for the calculation of each mean BP estimate and the absolute values of $\Delta P_{\mathrm{s}}$,

Table 1. - Sleep and blood pressure characteristics in the studied subjects

\begin{tabular}{lccc}
\hline & Controls & OSAS & p-value \\
\hline TIB min & $390 \pm 48$ & $394 \pm 52$ & NS \\
TST min & $301 \pm 55$ & $325 \pm 52$ & NS \\
Sleep efficiency \% & $76.9 \pm 6.9$ & $82.6 \pm 6.4$ & $<0.05$ \\
AHI & $2.5 \pm 2.4$ & $81.7 \pm 22.8$ & $<0.001$ \\
AH/TIB n.h ${ }^{-1}$ & $1.9 \pm 1.9$ & $67.7 \pm 19.9$ & $<0.001$ \\
Mean real $P$ s mmHg & $118 \pm 20$ & $140 \pm 15$ & $<0.005$ \\
Mean real $P$ d $\mathrm{mmHg}$ & $63 \pm 10$ & $78 \pm 11$ & $<0.002$ \\
Beat-by-beat $P$ s CV & $0.06 \pm 0.01$ & $0.16 \pm 0.02$ & $<0.001$ \\
Beat-by-beat $P$ d CV & $0.07 \pm 0.01$ & $0.20 \pm 0.03$ & $<0.001$ \\
\hline
\end{tabular}

Data are shown as mean \pm SD. OSAS: obstructive sleep apnoea syndrome; TIB: time in bed; TST: total sleep time; AHI: apnoea/ hypopnoea index; AH/TIB: apnoeas+hypopnoeas per hour of TIB; Mean real $P_{\mathrm{s}}$ and $P \mathrm{~d}$ : mean systolic and diastolic blood pressure calculated on all nocturnal heart beats; $\mathrm{CV}$ : coefficient of variation. NS: not significant. 
Table 2. - Characteristics of blood pressure samplings

\begin{tabular}{|c|c|c|c|c|}
\hline \multirow{2}{*}{ Sampling interval min } & \multicolumn{2}{|c|}{ No. of $P \mathrm{~s}$ or $P \mathrm{~d}$ values averaged $/$ mean $P \mathrm{~s}$ or $P \mathrm{~d}$ estimate } & \multicolumn{2}{|c|}{ No. of estimated $P$ s and $P$ d means/subject } \\
\hline & Controls & OSAS & Controls & OSAS \\
\hline 5 & $69.2(60-83)$ & $71.2(60-91)$ & $4.1(2-5)$ & $4.6(2-5)$ \\
\hline 10 & $34.3(30-41)$ & $35.5(30-46)$ & $8.6(3-10)$ & $9.3(6-10)$ \\
\hline 15 & $24.2(20-31)$ & $24.0(20-31)$ & $11.0(8-15)$ & $12.8(4-15)$ \\
\hline 20 & $17.1(15-21)$ & $17.5(15-23)$ & $16.7(10-20)$ & $17.6(10-20)$ \\
\hline 30 & $11.8(10-15)$ & $11.8(10-15)$ & $22.6(16-30)$ & $25.1(13-30)$ \\
\hline
\end{tabular}

Data presented as mean (range). $P_{\mathrm{s}}$ : systolic pressure; $P$ d: diastolic pressure; OSAS: obstructive sleep apnoea syndrome.

$\Delta P \mathrm{~d}, \Delta P_{\mathrm{s}} \%$ or $\Delta P \mathrm{~d} \%$ for any tested sampling interval in any subject.

Mean $\Delta P \mathrm{~s}$ and $\Delta P \mathrm{~d}$ values for each sampling interval in OSAS and control subjects are shown in figure 2 . Within both groups, mean $\Delta P \mathrm{~s}$ and, $\Delta P \mathrm{~d}$ values were similar at all sampling rates, and their scatter increased progressively as the sampling interval increased. However, variance of both $\Delta P \mathrm{~s}$ and $\Delta P \mathrm{~d}$ was significantly higher in OSAS than in controls for each sampling interval $(\mathrm{p}<0.001)$.

Figure 3 shows the frequency of $\Delta P_{\mathrm{s}} \%$ and $\Delta P_{\mathrm{d}} \%$ that did not exceed a range of $\pm 2.5,5,7.5,10$, and $12.5 \%$ at each sampling interval. In the control group, a sampling time of 30 min was sufficient to obtain $>95 \%$ of both mean nocturnal $P_{\mathrm{s}}$ and $P \mathrm{~d}$ estimates with an error $\left(\Delta P_{\mathrm{s}} \%\right.$ and $\Delta P \mathrm{~d} \%)$ in the range $\pm 5 \%$. Conversely, in the OSAS group the sampling interval had to decrease to at least 10 min for a comparable level of accuracy; however, a
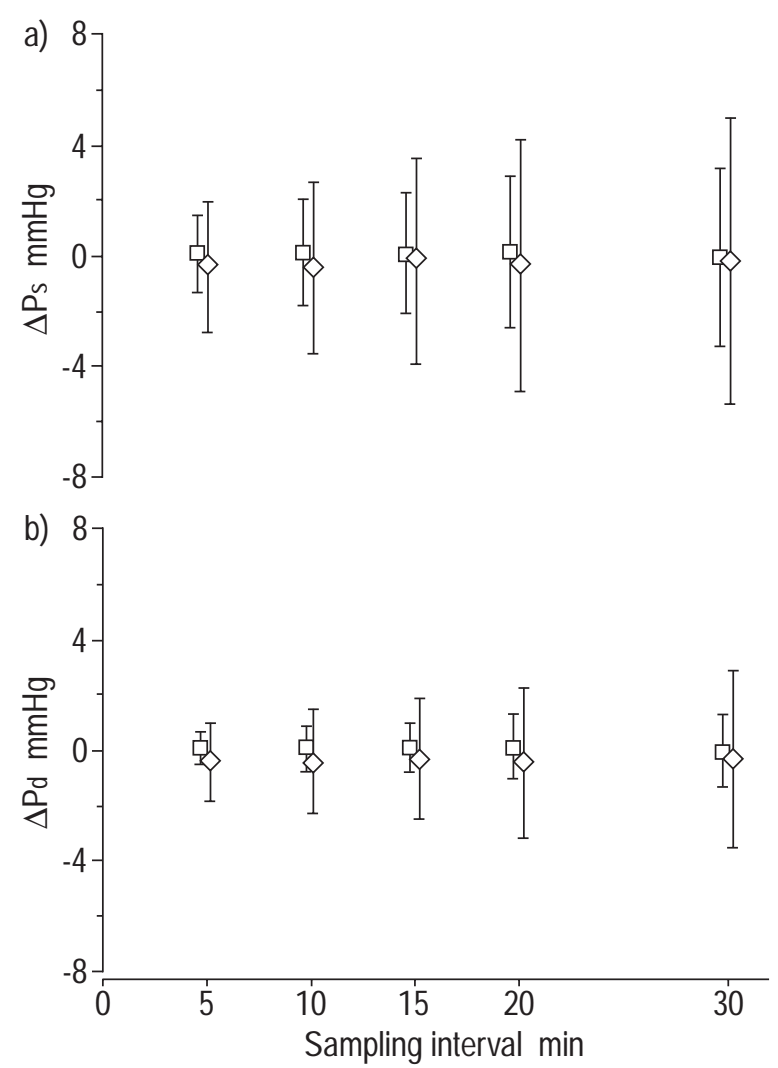

Fig. 2. - Means and SD of a) $\Delta P$ s and b) $\Delta P$ d values in control ( $\square$ ) and obstructive sleep apnoea $(\diamond)$ subjects for each sampling interval. $\Delta P \mathrm{~s}$ and $\Delta P \mathrm{~d}$ : differences in $\mathrm{mmHg}$ between the mean nocturnal blood pressure values, respectively systolic and diastolic, obtained from sampled single heart beats and from all heart beats. sampling interval of $30 \mathrm{~min}$ was enough even in this group to obtain $>95 \%$ of both mean nocturnal $P$ s and $P$ d estimates with an error in the range $\pm 10 \%$.

In the OSAS group, neither AHI nor AH/TIB were correlated to beat-by-beat $P \mathrm{~s}$ or $P$ d coefficients of variation; similarly, they were not correlated to mean $\Delta P \mathrm{~s}, \Delta P \mathrm{~d}$, $\Delta P_{\mathrm{s}} \%$ or $\Delta P \mathrm{~d} \%$; expressed in absolute values.

\section{Discussion}

In non-OSAS subjects it has already been shown that BP sampling intervals of 30, or, sometimes, even $60 \mathrm{~min}$ are accurate enough to evaluate mean nocturnal BP values $[1-3,18]$. In OSAS subjects, despite the well-known fact that BP during sleep has a much greater short-term variability than in normal subjects [14], similar intervals between nocturnal BP measurements have often been used when performing ABPM [7-11, 19-21], but the expected accuracy of the estimate of mean BP was not evaluated before this study.

The present aim was to evaluate to what extent ABPM may provide erroneous mean BP estimates due to an insufficient sampling rate. Rather than fully validating the use of ABPM in OSAS, this study regarded one of the aspects that influences the accuracy of ABPM in the assessment of mean BP, i.e. the length of intervals between BP measurements, in subjects with and without OSAS. In each of 18 OSAS and 9 control subjects several mean values of nocturnal BP were calculated, by sampling heart beats at different rates, and by changing repeatedly the heart beat from which sampling was started for each sampling rate. In this way the distribution of the difference between mean values obtained from sampled heart beats and from beat-to-beat measurements could be evaluated. This allowed to assess the probability to obtain values differing by more than predetermined thresholds from the real ones at different sampling rates. Such probability was found much higher in OSAS than in normal subjects.

In both OSAS and control subjects most mean BP estimates were close to mean real BP at all the tested sampling rates, but a very important difference between patients and controls was found in the scatter of the differences between estimated and real mean BP values: standard deviations of both $\Delta P \mathrm{~s}$ and $\Delta P \mathrm{~d}$ increased progressively as sampling interval was increased, as expected, while they remained about two-fold higher in the patients than in the controls at all the sampling intervals. This may have resulted from the higher BP variability in the patients. Since every apnoeic cycle is associated with a transient BP increase, the accuracy of mean nocturnal BP estimate could have been proportional to the rate of apnoeic and 

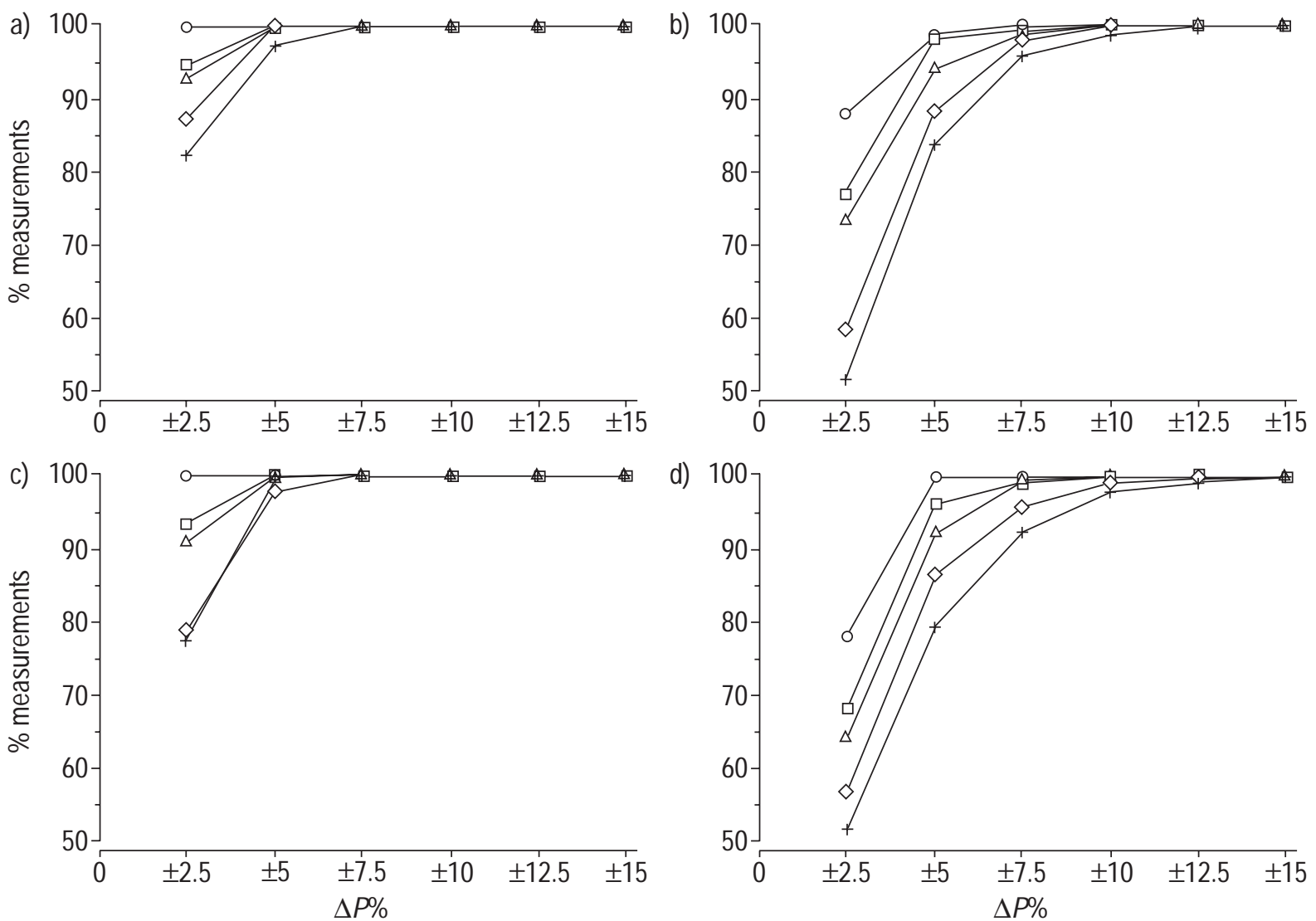

Fig. 3. - Percentage of measurements with $\Delta$ systolic pressure $(P \mathrm{~s}) \%(\mathrm{a}, \mathrm{b})$ and $\Delta$ diastolic pressure $(P \mathrm{~d}) \%(\mathrm{c}, \mathrm{d})$ not exceeding $\pm 2.5,5,7.5,10$, and $12.5 \%$. a) and c) in the whole sample of controls; b) and d) in the whole sample of obstructive sleep apnoea subjects. $\Delta P \mathrm{~s} \%$ and $\Delta P \mathrm{~d} \%=\mathrm{differences}$ between the mean nocturnal blood pressure values, respectively systolic and diastolic, obtained from sampled single heart beats and from all heart beats, expressed as percentage of the mean values calculated on all heart beats. Different symbols indicate different sampling intervals; $\bigcirc: 5$ min; $\square: 10$ min; $\triangle: 15 \mathrm{~min} ; \diamond: 20 \mathrm{~min} ;+: 30 \mathrm{~min}$.

hypopnoeic episodes. In our group of patients, despite AHI and AH/TIB showed a large range of values, neither of them was correlated to $P \mathrm{~s}$ or $P$ d coefficient of variation; as a consequence, they were not correlated to the accuracy of $\mathrm{BP}$ estimates (evaluated by $\Delta P \mathrm{~s}, \Delta P \mathrm{~d}, \Delta P_{\mathrm{s}} \%$ and $\Delta P \mathrm{~d} \%$ ). These findings may be explained by the fact that BP variability does not depend only on the number, but also on the amplitude of BP swings. In fact, a large intersubject variability was shown in the amplitude of BP swings associated with apnoeic episodes, possibly related to factors such as age or $\mathrm{Sa}_{\mathrm{a}} \mathrm{O}_{2}$ fall [22].

The number of points averaged to calculate mean nocturnal BP estimates was similar among control and OSAS subjects. In both groups, within each sampling interval taken in consideration, no increase in the difference between estimated and real mean nocturnal BP was observed as the number of points averaged to calculate mean BP estimate decreased. Therefore, the threshold of number of BP points selected to accept mean BP estimates was appropriate to give an indication of the effect of various lengths between BP measurements, such as those used during ABPM, on the accuracy of nocturnal BP estimate in OSAS and non-OSAS subjects. Furthermore, even in the assessment of mean BP by ABPM occasional failures in BP measurements often occur: thus, the number of points we sampled for mean BP estimates at each sampling interval could reflect the number of measurements actually per- formed by ABPM when the same interval between measurements is set.

Two points about differences between the two groups deserve some discussion. First, BP was higher in the OSAS group: however, the larger scatter in mean BP estimates among the OSAS subjects was evident both when BP differences were evaluated in $\mathrm{mmHg}$, and as percentage of the mean real values; this suggests that BP levels did not influence the results. Second, sleep efficiency was lower in the control group. More prolonged wakefulness periods may have caused an increase in BP variability, since in normal subjects BP variability is higher during wakefulness than during sleep [23]; a similar effect may have followed more frequent shifts between sleep and wakefulness; the increase in BP variability, in turn, may have decreased the accuracy of BP estimates. Therefore, for similar values of sleep efficiencies in normal and OSAS subjects, differences in mean nocturnal BP estimate accuracies could be slightly larger, but not smaller, than the ones found: this would not modify the conclusions about a much greater probability of inaccurate BP estimates by ABPM in OSAS than in normal subjects.

Several studies on 24-h BP by ABPM in OSAS have recently been published. Some of them were aimed at assessing the effect of long-term treatment on BP levels [6-11]; in only one of these studies was a 15 min sampling time used for nocturnal BP [6], while in the others a 
30 -min $[7,9-11]$ or 60 -min sampling time was chosen [8, $21]$. Inconsistent results have been reported, not only from paper to paper but also among patients of single studies. Other studies investigated how often OSAS patients could be considered "nondippers" [24]; also in this case BP sampling intervals of either 15 [25, 26], 30 $[11,19,20]$, or $60 \mathrm{~min}$ [21] were used. Although the differences among the findings of the studies did not appear related to the chosen sampling rate, a too long interval between measurements may have contributed to the inconsistencies of some results.

Before considering any estimated value as acceptable, it should be kept in mind what degree of accuracy is expected from the measurement, and what is the probability that the measurement reaches the required accuracy. For example, if an error within $10 \%$ mean nocturnal BP in a single night is required with a $\geq 95 \%$ probability, both in normal and in OSAS subjects a 30-min sampling interval would be accurate enough. However, if an error within $10 \%$ is required in the assessment of the difference between mean nocturnal BP of two different nights, then the highest error within each nocturnal measurement must be kept within $\pm 5 \%$, because the variance of the difference between two random variables is equal to the sum of the variances of each variable; in order to have the same probability that this threshold is not overcome, in normal subjects the 30-min sampling interval would remain accurate enough, providing further evidence that a 30 min interval between nocturnal measurements is adequate for non-OSAS subjects, while in the OSAS subjects the sampling interval should decrease to at least $10 \mathrm{~min}$.

The authors are aware that too frequent BP measurements during ABPM may cause sleep disruption [27-30] that, according to some Authors, in normal subjects [29] as well as in snorers [30] may cause an increase in BP with an alteration particularly in the evaluation of the systolic values. According to the following data collected by the authors, consequences of cuff inflations on sleep structure and BP behaviour may be much less important in severe OSAS subjects than those reported in nonOSAS subjects. In five OSAS subjects (age 40-57 yrs, AHI 71-83) ABPM measurements were taken every 15 min during polysomnography. A small hose was placed under the cuff and connected to a pressure transducer: the signal recorded during compression and decompression of the hose allowed to exactly identify when the cuff was inflated and deflated. Because of the effect of apnoeic cycles in causing arousal, the independent arousing effect of cuff inflation (that usually lasted more than one apnoea with the following arousal) was difficult to detect. As ABPM-induced arousals the occurrence, during or immediately after the BP measurement by the ABPM device, of: a) a change in breathing pattern due to prolonged awakening; b) a shortening of apnoea following cuff inflation with a smaller $\mathrm{Sa}_{\mathrm{a}} \mathrm{O}_{2}$ fall compared to the preceding apnoeic cycles $\left(\Delta \mathrm{Sa}_{\mathrm{a}, \mathrm{O}_{2}}>3 \%\right)$ were considered. Altogether, $141 \mathrm{BP}$ measurements were automatically performed. Among them, 19 occurred during wakefulness and a stable breathing pattern. Of the remaining 122 measurements, $20.5 \%$, (range per subject: $16-32 \%$ ) were classified as contributing to the occurrence of arousals or awakenings: all except two, were identified just by small reductions in $\mathrm{Sa}_{\mathrm{a}} \mathrm{O}_{2}$ falls, while apnoeas continued to recur. The much lower rate of cuff inflation-related arousals in these subjects than in those previously studied $[27,29,30]$ may be interpreted as a consequence of a high resistance to arousing stimuli in severe OSAS, due to a high degree of sleepiness. Therefore, despite the objective difficulty in assessing the arousing effect of cuff inflations when apnoeas recur continuously during sleep, the data indicate that the frequency of BP measurements by ABPM in severe OSAS subjects should be kept higher than in normal subjects, and that the results of this study can be taken in consideration before settling the interval between BP measurements during ABPM in these subjects.

\section{Conclusions}

Sampling interval heavily affects the probability to obtain accurate estimates of mean nocturnal blood pressure values, especially in obstructive sleep apnoea syndrome. In severe obstructive sleep apnoea syndrome, an accuracy in mean nocturnal blood pressure evaluation similar to normal subjects requires a shorter blood pressure sampling interval than in normal subjects. The selection of an appropriate sampling interval should be based on the degree of accuracy that is required in the assessment of blood pressure in each subject.

\section{References}

1. Di Rienzo M, Grassi G, Gregorini L, Pedotto A, Mancia G. Continuous vs intermittent blood pressure measurements in estimating 24 hours average blood pressure. Hypertens 1983; 5: 264-269.

2. Mancia G, Parati G, Pomidossi G, Di Rienzo M. Validity and usefulness of non-invasive ambulatory blood pressure monitoring. J Hypertens 1985; 3: S5-S11.

3. Schillaci G, Verdecchia P, Zampi I, Battistelli M, Bartoccini C, Porcellati C. Non-invasive ambulatory blood pressure monitoring during the night: randomised comparison of different reading intervals. J Hum Hypertens 1994; 8: 23-27.

4. Bonsignore MR, Marrone O, Insalaco G, Bonsignore G. The cardiovascular effect of obstructive sleep apnoeas: analysis of pathogenic mechanisms. Eur Respir J 1994; 7: 786-805.

5. Mayer J, Becker H, Brandenburg U, Penzel T, Peter JH, von Wichert P. Blood pressure and sleep apnoea: results of long-term nasal continuous positive airway pressure therapy. Cardiol 1991; 79: 84-92.

6. Wilcox I, Grunstein RR, Hedner JA, et al. Effect of nasal continuous positive airway pressure during sleep on 24hour blood pressure in obstructive sleep apnoea. Sleep 1993; 16: 539-544.

7. Suzuki M, Otsuka K, Guilleminault C. Long-term nasal continuous positive airway pressure administration can normalize hypertension in obstructive sleep apnoea patients. Sleep 1993; 16: 545-549.

8. Davies RJO, Crosby J, Prothero A, Stradling JR. Ambulatory blood pressure and left ventricular hypertrophy in subjects with untreated obstructive sleep apnoea and snoring, compared with matched control subjects, and their response treatment. Clin Sci 1994; 86: 417-424.

9. Hedner J, Darpö B, Ejnell H, Caidahl K. Reduction in sympathetic activity after long-term CPAP treatment in 
sleep apnoea: cardiovascular implications. Eur Respir $J$ 1995; 8: 222-229.

10. Saaralainen S, Hasan J, Siitonen S, Seppäla E. Effect of nasal CPAP treatment on plasma volume, aldosterone and 24-h blood pressure in obstructive sleep apnoea. J Sleep Res 1996; 5: 181-185.

11. Engleman HM, Gough K, Martin SE, Kingshott RN, Padfield PL, Douglas NJ. Ambulatory blood pressure on and off continuous positive airway pressure therapy for the sleep apnoea/hypopnoea syndrome: effects; in "nondippers". Sleep 1996; 19: 378-381.

12. Partinen $\mathrm{M}$, Jamieson A, Guilleminault C. Long-term outcome for obstructive sleep apnoea syndrome patients. Mortality. Chest 1988; 94: 1200-1204.

13. Mayer J, Greb H, Herres T, et al. Nocturnal hemodynamics in patients with sleep apnoea. In: Peter JH, Podszus $\mathrm{T}$, von Wichert $\mathrm{P}$, eds. Sleep related disorders and internal diseases. Berlin-Heidelberg, Spinger-Verlag, 1987; pp. 315-320.

14. Leroy M, Van Surrell C, Pilliere R, et al. Short-term variability of blood pressure during sleep in snorers with or without apnoea. Hypertens 1996; 28: 937-943.

15. Imholz BPM, Wieling W, van Montfrans CA, Wesseling KH. Fifteen years experience with finger arterial pressure monitoring: assessment of the technology. Cardiov Res 1998; 38: 605-616.

16. Rechtschaffen A, Kales A. A manual of standardized terminology, techniques, and scoring system for sleep stages of human subjects. Los Angeles, CA, Brain Information Service/Brain Research Institute, University of California at Los Angeles, 1968.

17. Report of an American Academy of Sleep Medicine Task Force. Sleep related breathing disorders in adults: recommendations for syndrome definition and measurement techniques in clinical research. Sleep 1999; 22: 667-689.

18. Pickering TG. Clinical aspects of ambulatory monitoring and determinants of nominal ambulatory blood pressure. In: Ambulatory monitoring and blood pressure variability. London, Science Press, 1991; pp. 9.1-9.15.

19. Noda A, Okada T, Hatashi H, Yasuma F, Yokota M. 24- hour ambulatory blood pressure variability in obstructive sleep apnoea syndrome. Chest 1993; 103: 1343-1347.

20. Suzuki M, Guilleminault C, Otsuka K, Shiomi T. Blood pressure "dipping" and "non-dipping" in obstructive sleep apnoea syndrome patients. Sleep 1996; 19: 382-387.

21. Pelttari LH, Hietanen EK, Salo EK, Kataja MJ, Kantola IM. Little effect of ordinary antihypertensive therapy on nocturnal high blood pressure in patients with sleep disordered breathing. Am J Hypertens 1998; 11: 272-279.

22. Tun Y, Okabe S, Hida W, et al. Nocturnal blood pressure during apnoeic and ventilatory periods in patients with obstructive sleep apnoea. Eur Respir J 1999; 14: 12711277.

23. Van de Borne P, Nguyen H, Biston P, Linkowski P, Degaute J-P. Effects of wake and sleep stages on the 24-h autonomic control of blood pressure and heart rate in recumbent men. Am J Physiol 1994; 266: H548-H554.

24. Verdecchia P, Schillaci G, Porcellati C. Dippers versus non-dippers. J Hypertens 1991; 9: (Suppl. 8), S42-S44.

25. Wilcox I, Grustein RR, Collins FL, Doyle J, Kelley DT, Sullivan CE. Circadian rhythm of blood pressure in patients with obstructive sleep apnoea. Blood Pressure 1992; 1: 219-222.

26. Pankow W, Nabe B, Lies A, et al. Influence of sleep apnoea on 24-hour blood pressure. Chest 1997; 112: $1253-1258$.

27. Schwan A, Eriksson G. Effect on sleep - but not on blood pressure - of nocturnal non-invasive blood pressure monitoring. J Hypertens 1992; 10: 189-194.

28. Dimsdale JE, Coy TV, Ancoli-Israel S, Clausen J, Berry CC. The effect of blood pressure cuff inflation on sleep. A polysomnographic examination. Am J Hypertens 1993; 6: 888-891.

29. Davies RJO, Jenkins NE, Stradling Jr. Effect of measuring ambulatory blood pressure on sleep and on blood pressure during sleep. BMJ 1994; 308: 820-823.

30. Heude E, Bourgin P, Feigel P, Escourrou P. Ambulatory monitoring of blood pressure disturbs sleep and raises systolic pressure at night in patients suspected of suffering from sleep-disordered breathing. Clin Sci 1996; 91: 45-50. 